Article

\title{
Can We Rely on Flight Time to Measure Jumping Performance or Neuromuscular Fatigue-Overload in Professional Female Soccer Players?
}

\author{
Estrella Armada-Cortés ${ }^{1,2}$ [ , Javier Peláez Barrajón ${ }^{1}$, José Antonio Benítez-Muñoz ${ }^{3}$, \\ Enrique Navarro ${ }^{1}$ and Alejandro F. San Juan ${ }^{1, * \mathbb{D}}$ \\ 1 Department of Health and Human Performance, Sport Biomechanics Laboratory, \\ Facultad de Ciencias Actividad Física y Deporte-INEF, Universidad Politécnica de Madrid, 28040 Madrid, \\ Spain; cortesarmadaestrella@gmail.com (E.A.-C.); javi.pelaezb@gmail.com (J.P.B.); \\ enrique.navarro@upm.es (E.N.) \\ 2 Department of Physical Activity and Sports Science, Faculty of Sport Sciences, \\ University of Castilla-La Mancha, 45071 Toledo, Spain \\ 3 Department of Health and Human Performance, LFE Research Group, \\ Facultad de Ciencias Actividad Física y Deporte-INEF, Universidad Politécnica de Madrid, 28040 Madrid, \\ Spain; jbenitez020@gmail.com \\ * Correspondence: alejandro.sanjuan@upm.es
}

Received: 27 May 2020; Accepted: 24 June 2020; Published: 27 June 2020

check for updates

Featured Application: It is recommended that high-level sportswomen and men should be assessed with the force platform through the take-off velocity method in a vertical jump as gold standard technology to ensure correct performance and/or fatigue-overload control during the sport season.

\begin{abstract}
The main purpose of this study was to compare the validity of the take-off velocity method (TOV) measured with a force platform (FP) (gold standard) versus the flight time method (FT) in a vertical jump to measure jumping performance or neuromuscular fatigue-overload in professional female football players. For this purpose, we used a FP and a validated smartphone application (APP). A total of eight healthy professional female football players (aged $27.25 \pm 6.48$ years) participated in this study. All performed three valid trials of a countermovement jump and squat jump and were measured at the same time with the APP and the FP. The results show that there is a lack of validity and reliability between jump height $(\mathrm{JH})$ calculated through the TOV method with the FP and the FT method with the FP $(r=0.028, p>0.84$, intraclass correlation coefficient $(\mathrm{ICC})=-0.026)$ and between the JH measured with the FP through the TOV method and the APP with the FT method $(\mathrm{r}=0.116$, $p>0.43$, ICC $=-0.094(-0.314-0.157))$. A significant difference between the JH measured through the TOV with the FP versus the APP $(p<0.05)$, and a trend between the JH obtained with the FP through the TOV and the FT $(p=0.052)$ is also shown. Finally, the JH with the FP through the FT and the APP did not differ $(p>0.05)$. The eta-squared of the one-way ANOVA was $\eta 2=0.085$. It seems that only the TOV measured with a FP could guarantee the accuracy of the jump test in SJ+CMJ and SJ, so it is recommended that high-level sportswomen and men should be assessed with the FP through TOV as gold standard technology to ensure correct performance and/or fatigue-overload control during the sport season.
\end{abstract}

Keywords: vertical jump; flight time; take-off velocity; fatigue; muscle overload; performance; force platform; smartphone application; APP; female soccer 


\section{Introduction}

The vertical jump (VJ) is one of the most widely used performance tests. Its popularity is due both to its simplicity and effectiveness [1], and to the similarity between its movement patterns and sports, explosiveness, speed, and intensity [2,3]. Moreover, it is a useful tool to assess physical fitness in a healthy population (i.e., children, adults, and elderly people) [4-6], and to control an adequate musculoskeletal injury recovery [7]. There are different jump protocols; two of the most frequently used are: (1) The squat jump (SJ), which provides a basic leg strength parameter and is described by some authors as pure concentric [3]; (2) the counter movement jump (CMJ) adds an elastic-reactive component of the subject. It is estimated that the CMJ value can be up to $25 \%$ higher than the $\mathrm{SJ}$ in athletes [8]. Their performance has been used to: (1) monitor the positive effects of strength, plyometric, resistance, and speed training; and (2) control mechanical and neuromuscular fatigue status in individual and team sports [9]. Several researchers have found that CMJ performance is an interesting objective marker of fatigue and overcompensation for athlete performance [9], being one of the factors related to the high incidence of injuries (e.g., muscle overload) in the lower limb muscles $[10,11]$. Thus, a relationship has been observed between height loss in the CMJ and metabolic markers such as lactate or ammonium in the sprint [12], and after a Wingate test [13]. This suggests that through decreases in the CMJ's mechanical variables such as jump height $(\mathrm{JH})$, it should be possible to estimate the metabolic stress, neuromuscular fatigue, and overload of the subject $[5,12,14]$.

Specific performance factors for both the CMJ and SJ include a number of different kinematic and kinetic variables. Some of these variables are more sensitive than others to determine an athlete's neuromuscular status and may depends of the age and level of the subjects: $\mathrm{JH}$, power, velocity and force (peak, relative peak, and mean of the last three), rate of force development (RFD), and mean impulse [9]. In addition, it has been proven that it is better to average the number of jumps executed than to choose the one that obtained the maximum height [9].

To obtain the height value of the jump we can use two variables: the take-off velocity (TOV) or the flight time (FT) $[15,16]$ :

1. The TOV is the vertical velocity of the center of mass at takeoff. The height value of the jump will be the result of velocity at takeoff squared divided by twice the gravity:

$$
\left(h=\frac{V^{2}}{2 g}\right)
$$

2. The FT is the time period between takeoff and land contact (i.e., the subject is on the air, without land contact). The height value of the jump will be the result of the gravity value multiplied by FT squared, divided by eight:

$$
\left(h=\frac{g t^{2}}{8}\right)
$$

There are many tools used to evaluate the performance of the jump (e.g., force platforms (FP), contact mats, video analysis through a smartphone applications (APPs), accelerometers, infrared systems, Vertec Vertical Jump Measuring Device). Most devices use the FT to calculate the JH [17]. However, to measure it in an accurate manner, the height of the center of mass at takeoff and landing has to be the same. Any difference between the athlete's takeoff and landing center of mass position (e.g., different joint position of ankle, knee, or hip) could increase or decrease the FT, and then change the result of the JH estimation [15,17]. Considering these technical implications, TOV has been suggested as the more suitable method to evaluate the JH [18]. If the TOV can be measured easily by a FP, then it is considered the gold standard for measuring the height of the VJ $[19,20]$. A FP can measure and use either the FT or the TOV methodology, the latter being the most accurate method for determining the height of the VJ [19]. 
The need to quantitatively evaluate the performance of the athlete's $\mathrm{JH}$ has promoted the emergence of APPs for this purpose. Some authors have reported that these APPs have a high reliability and accuracy in their measures [21,22], while others qualify their results as equivocal, so it is not yet clear what is the ideal methodology to use [23].

Although the VJ and its performance can be extrapolated to any sport level, the greater the level the subjects have, the more correlation exists in its variables, so it is in high performance sport where it has more utility [24]. Moreover, the accuracy of the results is essential from a competitive point of view in high-level sports, for example in elite-cyclists improvements of around $0.6 \%$ are sufficient to make a difference [25].

Therefore, the main purpose of this study was to compare the validity of the TOV method using a FP versus the FT method using a FP and a smartphone application to measure jumping performance or neuromuscular fatigue-overload in professional female football players.

\section{Materials and Methods}

\subsection{Participant Selection: Inclusion and Exclusion Criteria}

A total of eight healthy professional female football players of the First and Second Spanish Football Divisions (Liga Iberdrola and Reto Iberbrola, respectively) (aged $27.25 \pm 6.48$ years; body mass $56.73 \pm 4.86 \mathrm{~kg}$; height $1.61 \pm 0.06 \mathrm{~m}$. Values expressed as mean \pm standard deviation) participated in this study.

Inclusion criteria were: (1) playing in a female professional soccer league; (2) not having suffered a musculoskeletal injury one year prior to the date of the protocol (i.e., checked through a previous exclusion questionnaire); (3) not presenting any cardiovascular, musculoskeletal, and/or neurological disease, nor previous ones that could affect participation in the study. Exclusion criteria were: (1) aged younger than 18 years; (2) having consumed any narcotic and/or psychotropic agents or drugs during the test. We have selected this specific study population to homogenize the level of the sample and to deepen the knowledge of women's professional soccer.

At the study outset, participants were informed of the study protocol, schedule, and nature of the exercises and tests to be performed before signing an informed consent form. The study protocol adhered to the tenets of the Declaration of Helsinki and was approved by the Ethics Committee of the Technical University of Madrid (Madrid, Spain).

\subsection{Experimental Design}

This study consisted of a single evaluation session (see Figure 1) in a laboratory in the same time frame to avoid the detrimental performance effects associated with circadian rhythm [26]. Subjects were required to avoid physically demanding activity in the $24 \mathrm{~h}$ prior to the session.

Three valid trials of a CMJ and SJ were conducted with each subject, with $60 \mathrm{~s}$ recovery between trials, and three minutes between each jump test (see Figure 1). All jumps were measured at the same time with the mobile application and on the FP.

The sessions began with a 10-min general warm up consisting of continuous running, specific running, joint mobility, and ballistic stretching exercises, followed by a specific pre-test warm-up where participants performed five SJs and CMJs with $30 \mathrm{~s}$ between each jump. After three minutes of rest, the participants started the jumping test. 


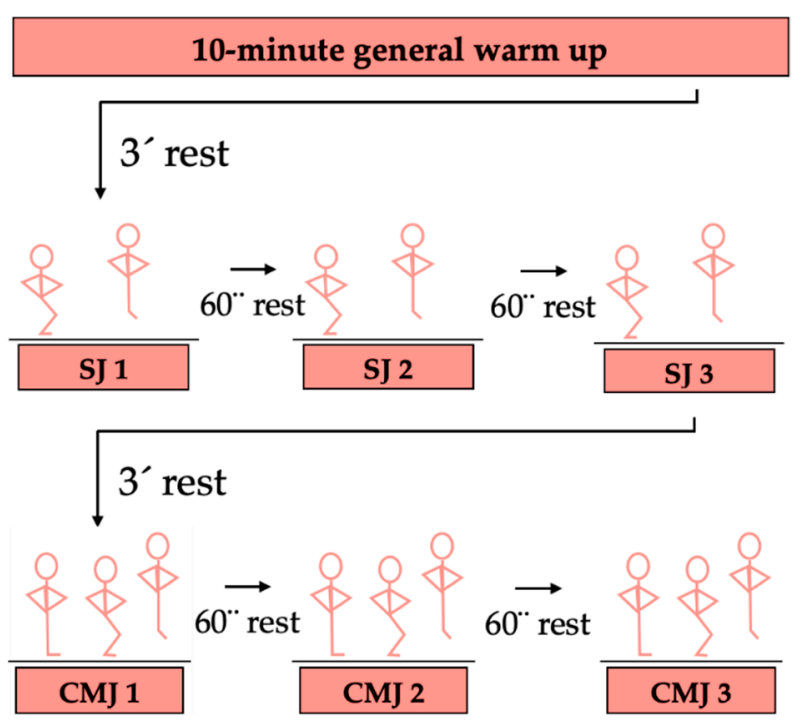

Figure 1. Experimental design. $\mathrm{CMJ}=$ countermovement jump test; $\mathrm{SJ}=$ squat jump.

\subsection{Vertical Jump Test}

The VJs included in the present study were the CMJ and SJ, which have been previously demonstrated to be reliable measures (intraclass correlation coefficient (ICC) $=0.97$ and 0.96 , respectively) [27]. All participants were completely familiarized with both techniques (i.e., they realized these tests during the soccer season). Moreover, they practice before the test during the specific warm-up (see warm-up description above). Participants were always instructed to jump as high as possible, keeping their hands on their hips and that their legs should remain straight during flight, making contact with the ground with the tips of their feet and knees extended [28]. In both modalities, the aim was to reach the maximum height by means of the jump; nevertheless, each modality of jump consists of different characteristics [29].

The CMJ (Figure 2a) initial position consists of a static standing position with hands on the hips. From this position, a continuous and fast triple hip, knee, and ankle flexion movement is executed until reaching $\approx 90^{\circ}$ of knee flexion, followed by the triple extension of the same joints in a fluid, fast, and continuous way [29]. In this type of VJ, there is a stretching-shortening cycle (SSC), which takes place during the consecutive eccentric, isometric, and concentric phases [29].

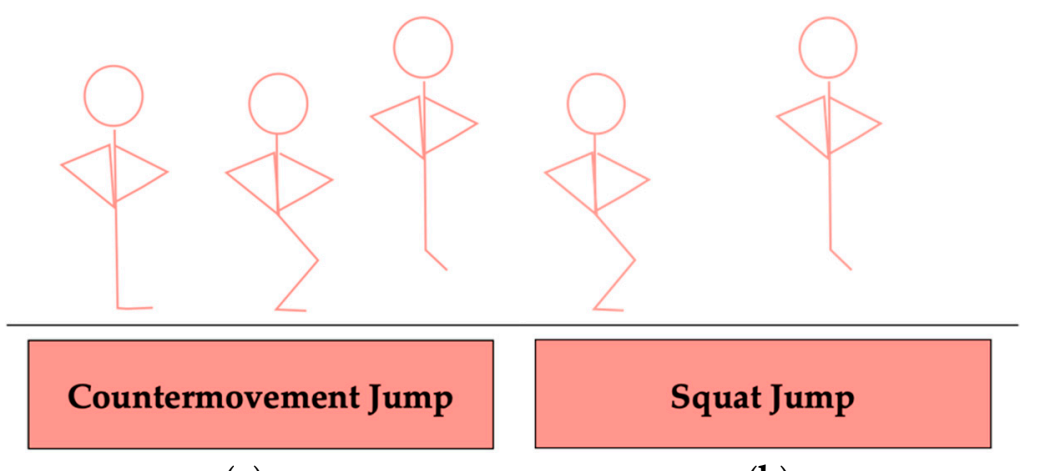

(a)

(b)

Figure 2. (a) Countermovement Jump; (b) Squat Jump.

The SJ (Figure 2b) only presents the concentric component. The initial position consists of maintaining the hips and knees flexed at $\approx 90^{\circ}$ for approximately four seconds to avoid countermovement and the elastic component. From this position, the concentric phase of the jump with an explosive extension of the lower limb joints is performed. All participants were asked to 
jump as high as possible and without performing a SSC [29]. The SJ forces before takeoff were checked on the FP to ensure that the participant did not perform countermovement.

For both jumps, participants were asked to take off and land at the same place to avoid lateral or horizontal displacement. Only successful trials were considered. Participants were asked to repeat the trial if a jump was incorrectly performed.

\subsection{Instruments}

\subsubsection{Force Platform}

Two $600 \mathrm{~mm} \times 400 \mathrm{~mm}$ piezoelectric platforms (Type 9286AA; Kistler Instruments AG, Winterthur, Switzerland) mounted together on the floor according to the manufacturer's instructions, were used in this experiment. The force sensors of these platforms were constituted by piezoelectric transducers $5 \mathrm{kN}$, total max $20 \mathrm{kN}$. Data were recorded at a sampling frequency of $1000 \mathrm{~Hz}$. The FPs were connected to a portable computer with specific software, the Measurement, Analysis and Reporting Software "Kistler MARS" (Kistler Instruments AG, Winterthur, Switzerland).

\subsubsection{Smartphone Application}

The validated smartphone application used to measure the performance of the jump was My Jump2 (APP) [30] installed on a mobile phone (iPhone 6s Apple, Cupertino, CA, USA) at a sampling rate of $240 \mathrm{~Hz}$. My Jump was designed for analyzing VJ measuring the time (in ms) between two frames selected by the user and subsequently to calculate the JH using the equation based on FT. The position of the camera was always at $\sim 1.5 \mathrm{~m}$ from the middle line of the subject so that it was constantly aligned with the reference joint points.

After the recording, one researcher manually selected the frames in which the subject performed exactly the moments of take-off and landing. A second person was consulted in case of doubt when analyzing the jumps with the APP. The JH was calculated based on the FT between the selected take-off and landing frame, as previously described [30].

\subsection{Statistical Analysis}

The normality and homogeneity of the data were analyzed using the Shapiro-Wilk and Levene tests, respectively. The normality and homogeneity of the dependent variables was confirmed $(p>0.05)$; all of the data are provided as their means and standard deviations.

Various statistical analyses were used to prove the APP validity and reliability in comparison with the FP using FT and TOV to calculate JH. The Pearson correlation coefficient (r) was used to calculate the concurrent validity between the APP and the FP using different measurement methods.

To expound the magnitude of the relationship between height measurements by the APP and the FP, Cohen's convention was used. To measure reliability between the APP and the FP, the coefficient of variation (CV), an absolute agreement Intraclass Correlation Coefficient (ICC), and Cronbach's Alpha were used. The CV was used to observe the uniformity of the values with respect to the mean. A Bland-Altman plot was created to graphically represent the agreement between the APP's measured heights and FP FT measured heights.

The mean differences between the measurements for each jump (SJ and CMJ) and for both jumps together $(\mathrm{CMJ}+\mathrm{SJ})$ were calculated through a one-way ANOVA, and Tukey's post-hoc test was used to analyze pairwise comparisons between means. The standard error of estimate (SEE) was used to show the typical error in measurement. Significance was set at $p<0.05$. All statistical analyses were performed using the software package SPSS ${ }^{\circledR}$ version 25 (IBM Co., USA). 


\section{Results}

The coefficients of variation values were very small for the CMJs with the FP through the TOV, the FP through the FT and the APP $(2.82 \%, 3.56 \%$, and 3.55\%, respectively), and for the SJ $(12.44 \%$, $5.24 \%$, and $4.88 \%$, respectively).

The Pearson's correlation showed a poor relationship and a small reliability between the JH measured through the FT and the TOV, both measured with the FP ( $\mathrm{r}=0.028, p>0.84, \mathrm{ICC}=-0.026$, 95\% CI $=-0.276-0.24$ ) (Figure 3), and between the JH measured with the FP through the TOV and the $\operatorname{APP}(\mathrm{r}=-0.116, p>0.43, \mathrm{ICC}=-0.094,95 \% \mathrm{CI}=-0.314-0.157)$ (Figure 4$)$.

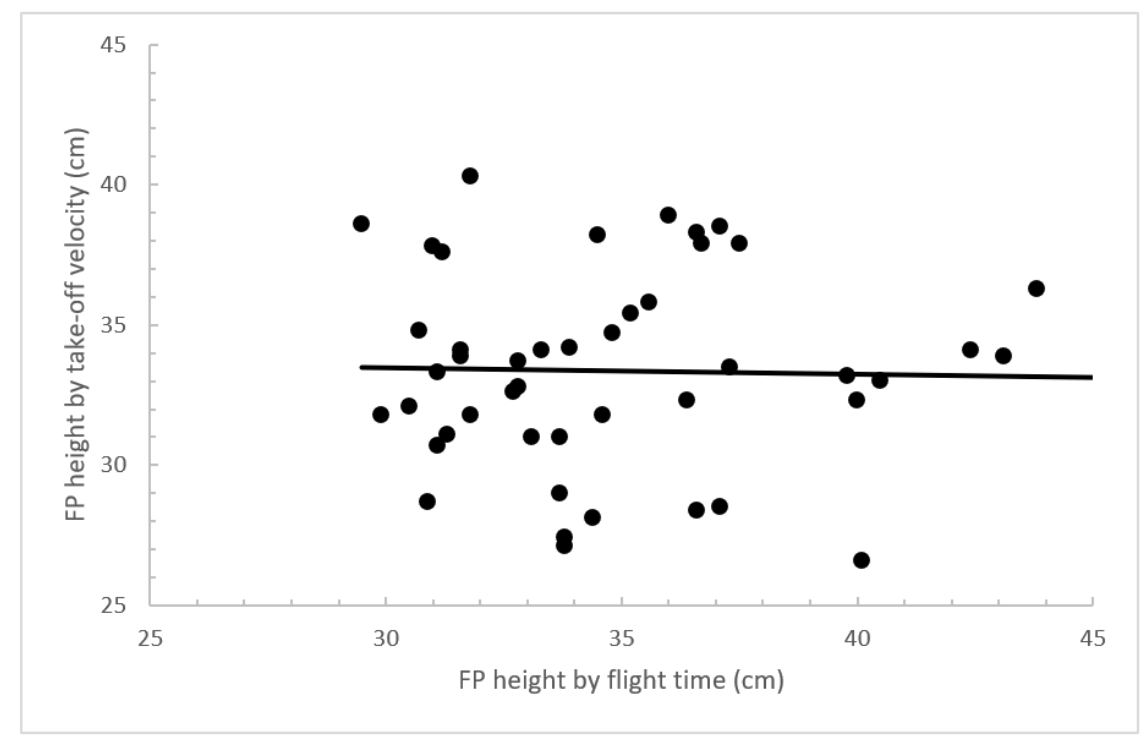

Figure 3. Pearson's correlation between jump heights measured by the FP using take-off velocity and the FP using flight time; FP, force platform; graph made by the authors.

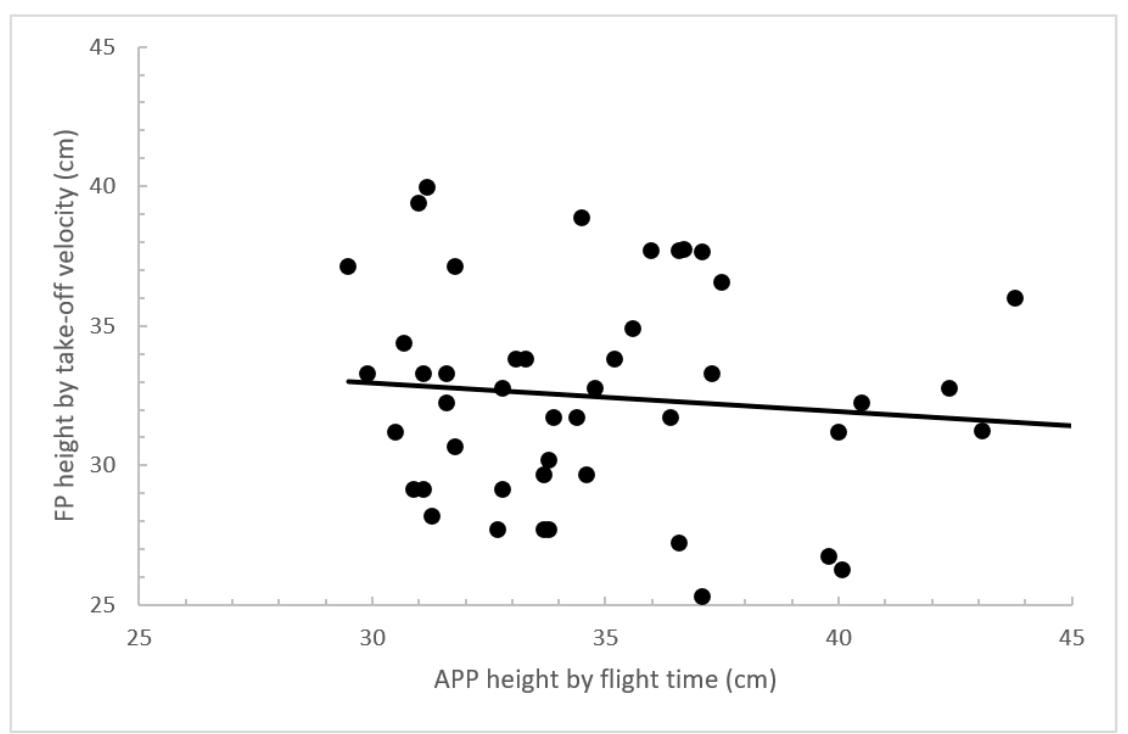

Figure 4. Pearson's correlation between jump heights measured by the FP using take-off velocity and the APP using flight time; FP, force platform; APP, My Jump App; graph made by the authors.

On the other hand, a high and significant relationship between the JH measured with the FP through the FT and the APP $(r=0.872, p<0.01)$ was found (Figure 5). Moreover, there was a very high agreement between these two variables as revealed by the ICC $=0.843(95 \% \mathrm{CI}=0.681-0.919)$ and Bland-Altman plots (Figure 6). 


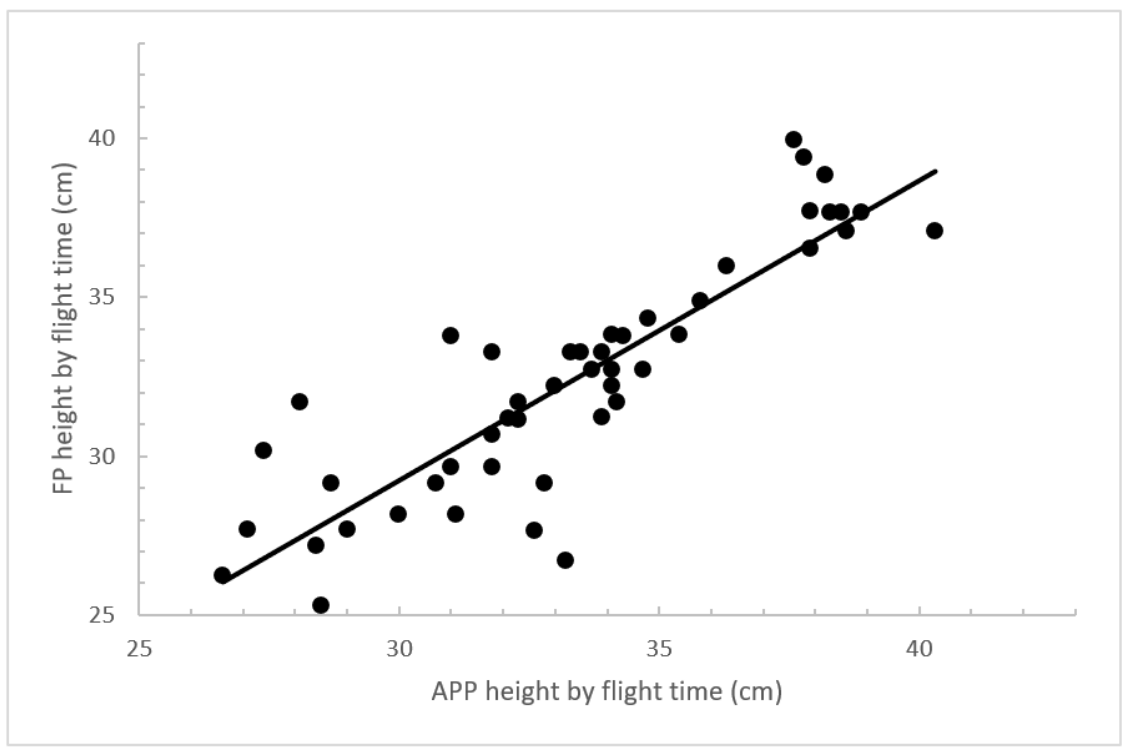

Figure 5. Pearson's correlation between jump heights measured by the FP using flight time and the APP using flight time; FP, force platform; APP, My Jump App; graph made by the authors.

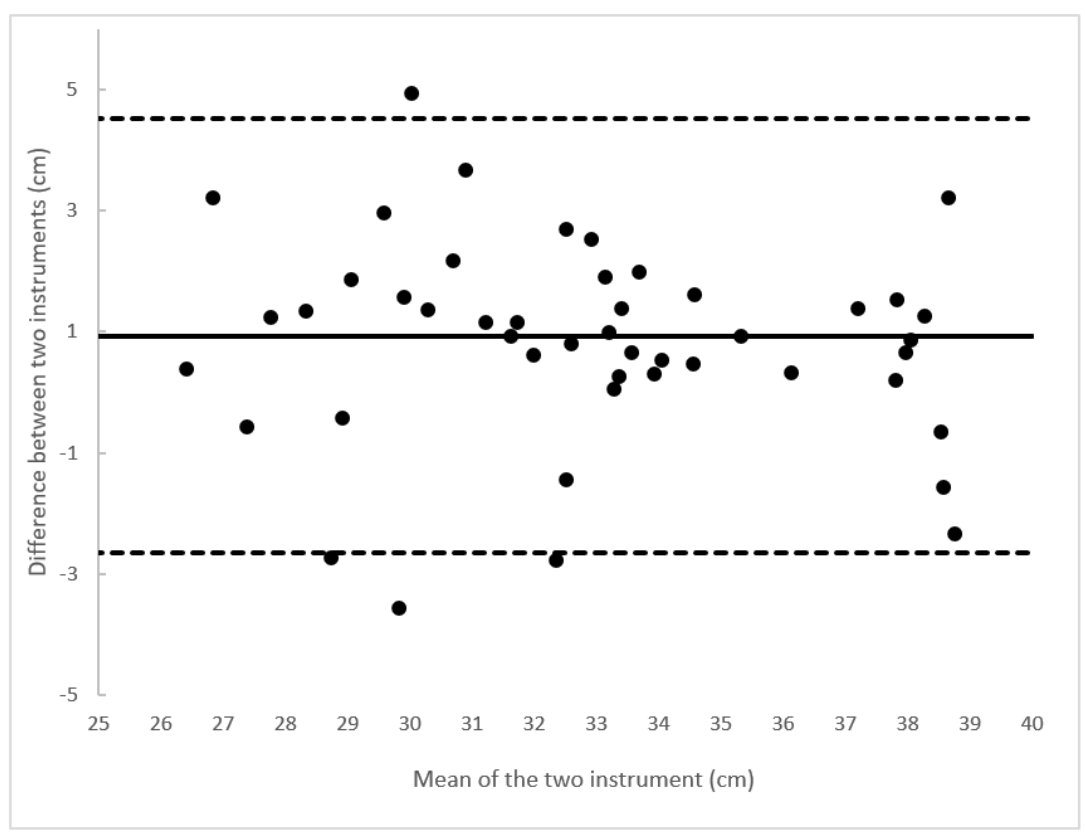

Figure 6. Bland-Altman plot between the FP using flight time and the APP using flight time to measure jump height. The central line represents the systematic bias between instruments, while the upper and the lower lines represent \pm 1.96 SD; FP, force platform; APP, My Jump App; graph made by the authors.

The one-way ANOVA shows a significant difference between the JH measured with the FP through the TOV versus the APP $(p=0.002)$, and a trend versus the FP through the FT $(p=0.052)$. Finally, the JH analyzed with the FP through the FT and the APP did not differ $(p=0.457)$ (Table 1$)$. The eta squared of the one-way ANOVA was $\eta 2=0.085$.

Specifically, in SJ, the one-way ANOVA shows a significant difference in JH measured with the FP through the TOV versus the FP and the APP both through the FT $(p<0.01)$. Finally, the JH analyzed with the FP through FT and the APP did not differ $(p=0.826)$ (Table 2). The eta squared of the one-way ANOVA used to compare SJ was $\eta 2=0.374$. 
Table 1. Values of the different methods for measuring jump height combining CMJ and SJ.

\begin{tabular}{ccccc}
\hline & Mean \pm SD & & Mean \pm SD & $p$ Value \\
\hline FP-TOV & $35.21 \pm 4.22$ & FP-FT & $33.36 \pm 3.48$ & 0.052 \\
FP-TOV & $35.21 \pm 4.22$ & APP & $32.43 \pm 3.77$ & $0.002 *$ \\
FP-FT & $33.36 \pm 3.48$ & APP & $32.43 \pm 3.77$ & 0.457 \\
\hline
\end{tabular}

Data are presented as mean \pm standard deviation. FP-TOV: Force Platform-Take-Off Velocity; FP-FT: Force PlatformFlight Time; APP: My Jump Application (flight time). * Significant difference.

Table 2. Values of the different methods for measuring SJ jump height.

\begin{tabular}{ccccc}
\hline & Mean \pm SD & & Mean \pm SD & $p$ Value \\
\hline FP-TOV & $37.23 \pm 4.68$ & FP-FT & $31.53 \pm 3.09$ & $0.000 *$ \\
FP-TOV & $37.23 \pm 4.68$ & APP & $30.89 \pm 3.33$ & $0.000 *$ \\
FP-FT & $31.53 \pm 3.09$ & APP & $30.89 \pm 3.33$ & 0.826 \\
\hline
\end{tabular}

Data are presented as mean \pm standard deviation. FP-TOV: Force Platform-Take-Off Velocity; FP-FT: Force PlatformFlight Time; APP: My Jump Application (flight time); SJ: squat jump. * Significant difference.

Lastly, in CMJ, the one-way ANOVA did not show significant differences between JH measured by the different methods $(p=0.07, \eta 2=0.073)$ (Table 3$)$.

Table 3. Values of the different methods for measuring CMJ jump height.

\begin{tabular}{ccccc}
\hline & Mean \pm SD & & Mean \pm SD & $p$ Value \\
\hline FP-TOV & $33.18 \pm 2.44$ & FP-FT & $35.19 \pm 2.87$ & 0.060 \\
FP-TOV & $33.18 \pm 2.44$ & APP & $33.96 \pm 3.60$ & 0.644 \\
FP-FT & $35.19 \pm 2.87$ & APP & $33.96 \pm 3.60$ & 0.336 \\
\hline
\end{tabular}

Data are presented as mean \pm standard deviation. FP-TOV: Force Platform-Take-Off Velocity; FP-FT: Force PlatformFlight Time; APP: My Jump Application (flight time); CMJ; countermovement jump.

\section{Discussion}

The main purpose of this study was to compare the validity of the TOV method using a FP versus the FT method using a FP and a smartphone application to measure jumping performance or neuromuscular fatigue-overload in professional female soccer players.

The major findings were the lack of reliability and validity between $\mathrm{JH}$ measured with the FP through the TOV and JH measured through the FT either by the FP $(r=0.028, p>0.84$, ICC $=-0.026$ $(-0.276-0.24))$ or the APP $(r=0.116, p>0.43$, ICC $=-0.094(-0.314-0.157))$. To our knowledge, this is the first study that has researched the JH of SJ and CMJ together (SJ + CMJ) with these technologies: FP trough the TOV and trough FT, and APP through FT. Two previous studies [20,31] have analyzed the SJ, CMJ and CMJ with arms (CMJA), together comparing JH with FP vs. contact mat (both through FT method) [20], and with FP (TOV and FT methods) vs. contact mat (FT method) [31]. This latter study [31] concluded that the TOV must be used instead of the FT as a more valid and accurate way to calculate the VJ performance. It provides a more sensitive approach to scientifically establishing jump determinants, greater sensitivity in analyzing changes in performance factors after training (i.e., overcompensation, overload), and greater accuracy in monitoring performance within the session (i.e., fatigue) $[18,19]$. Further, the mean comparison shows a significant difference between the JH measured with the FP through the TOV versus the APP $(p=0.002)$, and a trend versus the FP through the FT ( $p=0.052)$. To our knowledge, there is only one previous study that researched the differences between the FP through the TOV versus a smartphone application (FT method) [19], and it showed near perfect correlations (ICC $=0.996 ; p<0.001$ ). These differences with our results may be explain by some reasons: 1) In our study the subjects performed three different CMJs and SJs, while in the previous study the subjects performed five CMJs [19]; (2) The distance between the researcher and the subject was of $1 \mathrm{~m}$ in the previous study [19], while in our study it was of $1.5 \mathrm{~m}$; (3) In addition, rest 
between jumps was not specified in the previous study [19], so it is not possible to know if fatigue could have affected their results through the FT method (i.e., Different joint position between take-off and landing), whilst our subjects rested $60 \mathrm{~s}$ between trials and three minutes between each jump test. Despite these methodological differences, their results comparing the FP through the TOV versus a smartphone application (FT method) regarding only CMJ did not differ with our CMJ results. Based on the present study and the literature $[19,20]$, it seems that the flight time method, independent of the technology used (e.g., FP, contact mat, smartphone application), is an imprecise means of assessment.

On the other hand, there is a high correlation and good reliability between the JH SJ+CMJ measured through the FT with the FP and the APP $(r=0.872, p<0.01, \mathrm{ICC}=0.843, \mathrm{CV}=4.88 \%)$. These results are in accordance with other studies that compare the SJ+CMJ+CMJA height using a FP and a contact mat (both through FT) $(r=0.99, p<0.001$, ICC $=0.985, \mathrm{CV} \%=2.7)$ [21]. Additionally, the mean comparison of the present study shows that the JH analyzed with the FP through the FT and the APP did not differ $(p=0.457)$. Therefore, the APP and the contact mat seem to equal the accuracy of the FP with the FT method. However, it is important to highlight the fact that some studies compare the My Jump APP based on FT, with other instruments also based on FT (i.e., contact mat or FP through the FT) $[21,30,31]$, instead of comparing it with the FP through the TOV (gold standard) [16]. Therefore, all these technologies that use the FT method are not as precise as the TOV method. Bearing in mind that the most accurate and considered the gold standard method to measure the height of the jump is the FP based on the TOV $[19,20]$, these are the results that should be trusted.

Analyzing specifically the different jumps, it was found in SJ significant differences between the $\mathrm{JH}$ measured with the FP through the TOV and the FP and the APP through the FT $(p<0.01$ in both cases). However, we found no significant difference comparing the JH measured with the FP and the APP (both through the FT) $(p=0.826)$. Further, CMJ data showed no significant differences among the three methods (i.e., FP through the TOV versus the FP and the APP through the FT) $(p>0.05$ in both cases). Our results are in line with the only one previous study that researched the differences between the FP through the TOV versus a smartphone application (FT method) [19], and it showed near perfect correlations (ICC $=0.996 ; p<0.001$ ). Results of the reliability and validity obtained in the present study comparing the CMJ height through the FT with the FP and the APP $(\mathrm{r}=0.872, p<0.01$, ICC $=0.843$ and $\mathrm{CV}=4.88 \%$ ) are in accordance with the first validation study of My Jump App [30] that compare the CMJ height using a FP through FT ( $r=0.995, p<0.001$, ICC $=0.997, \mathrm{CV} \%=3.5)$, and other study that compare the CMJ height using a contact mat and the My Jump App (both through FT) $(r=0.999$, $p<0.001$, ICC $=0.948, C V \%=10.096$ ) [21]. Despite the differences between the sampling frequency of the devices (i.e., $\mathrm{FP} \approx 1000 \mathrm{~Hz}$ and the smartphone camera $\approx 240 \mathrm{~Hz}$ ), the height values obtained were very similar between the two methods, as reflected by the ICC.

The literature that evaluates the smartphone applications in this area has analyzed the CMJ as the only vertical jump option discarding the SJ $[19,21]$. However, with this study it has been demonstrated that the results of the CMJ cannot be extrapolated to the SJ. The height of the SJ and CMJ is defended by several authors as a method to detect fatigue and individualize load prescriptions, so the reduction of the height of the VJ can be interpreted as evidence of the deterioration of neuromuscular function (i.e., fatigue, neuromuscular overload) [12]. Its measurement could provide a relatively simple and accessible tool for quantifying the degree of neuromuscular, mechanical, and metabolic fatigue [5,12,32]. This fatigue can remain for up to $48-72 \mathrm{~h}$ after a high-intensity session [33]. It could therefore provide useful feedback to coaches to determine the impact of training loads on player recovery and performance [12]. The neuromuscular factor is considered the most influential factor in training and is also an intrinsic risk factor of an athlete's injury [34]. The lack of strength and poor coordination capacity are part of it [35]. Several studies have observed that after a fatiguing exercise bout and during the landing phase of different jump types, there is a change in the neuromuscular control strategies used by these subjects [36,37]. These motor control alterations are associated with different injury risk factors, such as reduced knee and hip flexion, increased knee valgus, increased ground reaction force, and greater stabilization time [37]. In high-level sports, measurement accuracy 
is critical in the assessment of performance and in the control and assimilation of training loads to avoid muscle-tendon overloads [25]. In addition, some authors recommend the evaluation of jumping performance, especially at high and elite levels [24]. In this sense and referring to the exclusive sample of this study, as they are professional female soccer players, these small variations described above should be considered and limited as far as possible. In our study, the differences in JH between the FP through the TOV versus through the FT was $5.23 \%(1.84 \mathrm{~cm})$ and the differences between the FP through the TOV versus the APP was $7.90 \%(2.78 \mathrm{~cm})$. The only previous study that researched the differences between the FP (TOV method) versus a smartphone application (FT method) [19], found a small overestimated JH obtained from the APP of $0.78 \%$ compared to the FP based on TOV [19]. This small difference $(0.78 \%)$ is more than ten times lower than the differences reported by us $(7.90 \%)$, more than six times the difference between FP-TOV vs. FT methods $(5.23 \%)$, and more than three times lower than the differences between the FP (FT method) and the APP $(2.8 \%)$. This latter difference reported in the present study between the FP (FT method) and the APP agree with other studies comparing the My Jump application and a FP (FT method) [30], and an infrared contact mat and a FP (both through the FT method) [19]. More research regarding the issue is needed to clarify if smartphone applications may replace a FP (TOV method) in professional environments where accuracy is a key factor (i.e., high-level sport, medical clinic).

Furthermore, we observed that the difference of JH between the FP and the APP both through the FT was smaller, only $2.8 \%(0.94 \mathrm{~cm})$. These latter results are in accordance with previous studies that observed differences in JH between an infrared contact mat and a FP, both through the FT of $\approx 2.5 \%$ $(1.06 \mathrm{~cm})$ [19], and between the My Jump application and a FP both through the FT $(1.1 \pm 0.5 \mathrm{~cm})$ [30]. These concordances are produced because they use the FT method (i.e., less accurate) to compare different technologies. When the FT method is used with any technology, even the FP, there are many possibilities to produce measurement errors because the takeoff and the landing positions are different (e.g., different degree of lower limbs joint/s flexion) [38]. Then, it seems recommendable that high-level sportswomen and men should be assessed with this gold standard technology and method (i.e., FP and TOV method) to ensure correct performance and/or overload control during the sport season.

\section{Conclusions}

The results of the present study showed that in female professional soccer players, there is a lack of validity and reliability between JH in SJ + CMJ and SJ calculated with the TOV method versus the FT method. It seems that only the TOV measured with a FP could guarantee the accuracy of the jump test.

In order to be able to generalize our conclusions to different athlete populations, future research is needed, aiming at comparing the validity and reliability between $\mathrm{JH}$ of different kind of jumps calculated with the TOV method versus the FT method on high-level and recreationally trained athletes from different sport modalities and both sexes.

Author Contributions: Conceptualization, E.A.-C., J.A.B.-M. and A.F.S.J.; methodology, E.A.-C., J.P.B., J.A.B.-M., E.N. and A.F.S.J.; validation, E.A.-C. and A.F.S.J.; formal analysis, E.A.-C., J.P.B. and A.F.S.J.; investigation, E.A.-C., J.P.B., J.A.B.-M. and A.F.S.J.; resources, E.A.-C., E.N. and A.F.S.J.; supervision, E.N. and A.F.S.J.; project administration, E.N. and A.F.S.J. All authors have read and agreed to the published version of the manuscript.

Funding: This research received no external funding.

Conflicts of Interest: The authors declare no conflicts of interest.

\section{References}

1. Cronin, J.; Gill, N.D. Fatigue Monitoring in High Performance Sport: A Survey of Current Trends. J. Aust. Strength Cond. 2012, 20, 12-23.

2. Yamauchi, J.; Ishii, N. Relations Between Force-Velocity Characteristics of the Knee-Hip Extension Movement and Vertical Jump Performance. J. Strength Cond. Res. 2007, 21, 703-709. [PubMed] 
3. Nagahara, R.; Naito, H.; Miyashiro, K.; Morin, J.B.; Zushi, K. Traditional and ankle-specific vertical jumps as strength-power indicators for maximal sprint acceleration. J. Sports Med. Phys. Fitness 2014, 54, 691-699. [PubMed]

4. Ramírez-Campillo, R.; Castillo, A.; de la Fuente, C.I.; Campos-Jara, C.; Andrade, D.C.; Álvarez, C.; Martinez, C.; Castro-Sepulveda, M.; Pereira, A.; Marques, M.C.; et al. High-speed resistance training is more effective than low-speed resistance training to increase functional capacity and muscle performance in older women. Exp. Gerontol. 2014, 58, 51-57. [CrossRef] [PubMed]

5. Maté-Muñoz, J.L.; Lougedo, J.H.; Garnacho-Castaño, M.V.; Veiga-Herreros, P.; Lozano-Estevan, M.D.C.; García-Fernández, P.; de Jesus, F.; Guodemar-Perez, J.; San Juan, A.F.; Dominguez, R. Effects of $\beta$-alanine supplementation during a 5-week strength training program: A randomized, controlled study. J. Int. Soc. Sports Nutr. 2018, 15, 19. [CrossRef]

6. Fernandez-Santos, J.R.; Ruiz, J.R.; Cohen, D.D.; Gonzalez-Montesinos, J.L.; Castro-Piñero, J. Reliability and Validity of Test to Assess Lower-Body Muscular Power in Children. J. Strength Cond. Res. 2015, 29, 2277-2285. [CrossRef]

7. Setuain, I.; Millor, N.; Alfaro, J.; Gorostiaga, E.; Izquierdo, M. Jumping performance differences among elite professional handball players with or without previous ACL reconstruction. J. Sports Med. Phys. Fitness 2015, 55, 1184-1192.

8. Sánchez, W.G.; Gómez, D.A.; Quiceno, H.B.; Alzate, S.J. Análisis comparativo intrasujeto en salto vertical 2d: Squat jump y counter-movement jump Resumen Introducción. VIREF Rev. Educ. Fís. 2016, 5, 1-17.

9. Gustavo, J.; Cronin, J.; Mezêncio, B.; Travis, D.; Mcguigan, M.; Tricoli, V.; Carlos Amadio, A.; Cerca Serrao, J. The countermovement jump to monitor neuromuscular status: A meta-analysis. J. Sci. Med. Sport 2017, 20, 397-402.

10. Greig, M.; Siegler, J.C. Soccer-specific fatigue and eccentric hamstrings muscle strength. J. Athl. Train. 2009, 44, 180-184. [CrossRef]

11. Science, E.; Kingdom, U.; Activity, P.; Kingdom, U. Effect of Timing of Eccentric Hamstring Strengthening Exercises During Soccer Training: Implications for Muscle Fatigability. J. Strength Cond. Res. 2009, 23, 1077-1083.

12. Jiménez-Reyes, P.; Pareja-Blanco, F.; Cuadrado-Peñafiel, V.; Ortega-Becerra, M.; Párraga, J.; González-Badillo, J.J. Jump height loss as an indicator of fatigue during sprint training. J. Sports Sci. 2018, 37, 414. [CrossRef] [PubMed]

13. Reeve, T.C.; Tyler, C.J. The validity of the smartjump contact mat. J. Strength Cond. Res. 2013, 27, 1597-1601. [CrossRef] [PubMed]

14. Aragón-Vargas, L. Evaluation of Four Vertical Jump Tests: Methodology, Reliability, Validity and accuracy. Meas. Phys. Educ. Exerc. Sci. 2000, 4, 215-228. [CrossRef]

15. Buckthorpe, M.; Morris, J.; Folland, J.P. Validity of vertical jump measurement devices. J. Sports Sci. 2012, 30, 63-69. [CrossRef] [PubMed]

16. Moir, G.L. Three different methods of calculating vertical jump height from force platform data in men and women. Meas. Phys. Educ. Exerc. Sci. 2008, 12, 207-218. [CrossRef]

17. Ferro, A.; Floría, P.; Villacieros, J.; Muñoz-lópez, A. Maximum velocity during loaded countermovement jumps obtained with an accelerometer, linear encoder and force platform: A comparison of technologies. J. Biomech. 2019, 95, 109281. [CrossRef]

18. Jiménez-Reyes, P.; Pareja-Blanco, F.; Rodríguez-Rosell, D.; Marques, M.C.; González-Badillo, J.J. Maximal velocity as a discriminating factor in the performance of loaded squat jumps. Int. J. Sports Physiol. Perform. 2016, 11, 227-234. [CrossRef]

19. Carlos-Vivas, J.; Martin-Martinez, J.P.; Hernandez-Mocholi, M.A.; Perez-Gomez, J. Validation of the iphone app using the force platform to estimate vertical jump height. J. Sports Med. Phys. Fitness 2018, 58, 227-232.

20. Glatthorn, J.F.; Gouge, S.; Nussbaumer, S.; Stauffacher, S.; Impellizzeri, F.M.; Maffiuletti, N.A. Validity and Reliability of Optojump Photoelectric Cells for Estimating Vertical Jump Height. J. Strength Cond. Res. 2011, 25, 556-560. [CrossRef]

21. Cruvinel-Cabral, R.M.; Oliveira-Silva, I.; Medeiros, A.R.; Claudino, J.G.; Jiménez-Reyes, P.; Boullosa, D.A. The validity and reliability of the "my Jump App" for measuring jump height of the elderly. PeerJ 2018, 6, e5804. [CrossRef] [PubMed] 
22. Brooks, E.R.; Benson, A.C.; Bruce, L.M. Novel technologies found to be valid and reliable for the measurement of vertical jump height with jump-and-reach testing. J. Strength Cond. Res. 2018, 32, 2038-2845. [CrossRef] [PubMed]

23. Leard, J.S.; Cirillo, M.A.; Katsnelson, E.; Kimiatek, D.A.; Miller, T.W.; Trebincevic, K.; Garbalosa, J.C. Validity of Two Alternative Systems for Measuring Vertical Jump Height. J. Strength Cond. Res. 2007, 21, 1296-1299. [PubMed]

24. Jiménez-Reyes, P.; Samozino, P.; García-Ramos, A.; Cuadrado-Peñafiel, V.; Brughelli, M.; Morin, J.-B. Relationship between vertical and horizontal force-velocity-power profiles in various sports and levels of practice. PeerJ 2018, 6, e5937. [CrossRef]

25. Paton, C.D.; Hopkins, W.G. Variation in performance of elite cyclists from race to race. Eur. J. Sport Sci. 2006, 6, 25-31. [CrossRef]

26. Drust, B.; Waterhouse, J.; Atkinson, G.; Edwards, B.; Reilly, T. Circadian Rhythms in Sports Performance-An Update; Chronobiology International; Taylor and Francis Inc.: Abingdon, UK, 2005; Volume 22, pp. $21-44$.

27. Markovic, G.; Dizdar, D.; Jukic, I.; Cardinale, M. Reliability and Factorial Validity of Squat and Countermovement Jump Tests. J. Strength Cond. Res. 2004, 18, 551-555.

28. Jiménez-Reyes, P.; Cuadrado-Peñafiel, V.; González-Badillo, J.; Alfonso El Sabio, U.X.; Jiménez Reyes Universidad Alfonso el Sabio, P.X. Analysis of Variables Measured in Vertical Jump Related to Athletic Performance and its Application to Training. Cult. Cienc. Deport. 2011, 6, 113-119.

29. Bosco, C. La Fuerza Muscular. Aspectos Metodológicos, 1st ed.; INDE, Ed.; INO Reproducciones, S.A.: Barcelona, Spain, 2000; pp. 177-187.

30. Balsalobre-Fernández, C.; Glaister, M.; Lockey, R.A. The validity and reliability of an iPhone app for measuring vertical jump performance. J. Sports Sci. 2015, 33, 1574-1579. [CrossRef]

31. Gallardo-Fuentes, F.; Gallardo-Fuentes, J.; Ramírez-Campillo, R.; Balsalobre-Fernández, C.; Martínez, C.; Caniuqueo, A.; Canas, R.; Banzer, W.; Loturco, I.; Nakamura, F.; et al. Intersession and intrasession reliability and validity of the my jump app for measuring different jump actions in trained male and female athletes. J. Strength Cond. Res. 2016, 30, 2049-2056. [CrossRef]

32. San Juan, A.F.; López-Samanes, Á.; Jodra, P.; Valenzuela, P.L.; Rueda, J.; Veiga-Herreros, P.; Perez-Lopez, A.; Dominguez, R. Caffeine supplementation improves anaerobic performance and neuromuscular effciency and fatigue in Olympic-level boxers. Nutrients 2019, 11, 2120. [CrossRef]

33. Hellsten-westing, Y.; Norman, B.; Balsom, P.D.; Sjodin, B. Decreased resting levels of adenine nucleotides in human skeletal muscle after high-intensity training. J. Appl. Phys. 1993, 74, 2523-2528. [CrossRef] [PubMed]

34. Griffin, L.Y.; Albohm, M.J.; Arendt, E.A.; Bahr, R.; Beynnon, B.D.; DeMaio, M.; Dick, R.W.; Engebretsen, L.; Garrett, W.E.; Hannafin, J.A.; et al. Understanding and preventing noncontact anterior cruciate ligament injuries: A review of the Hunt Valley II Meeting, January 2005. Am. J. Sports Med. 2006, 34, 1512-1532. [CrossRef] [PubMed]

35. Fort Vanmeerhaeghe, A.; Romero Rodriguez, D. Neuromuscular risk factors of sports injury. In Apunts Medicina de l'Esport; Elsevier España, S.L.: Cataluña, Spain, 2013; Volume 48, pp. 109-120.

36. Borotikar, B.S.; Newcomer, R.; Koppes, R.; McLean, S.G. Combined effects of fatigue and decision making on female lower limb landing postures: Central and peripheral contributions to ACL injury risk. Clin. Biomech. 2008, 23, 81-92. [CrossRef] [PubMed]

37. McLean, S.G.; Felin, R.E.; Suedekum, N.; Calabrese, G.; Passerallo, A.; Joy, S. Impact of fatigue on gender-based high-risk landing strategies. Med. Sci. Sports Exerc. 2007, 39, 502-514. [CrossRef] [PubMed]

38. Kibele, A. Possibilities and Limitations in the Biomechanical Analysis of Countermovement Jumps: A Methodological Study. J. Appl. Biomech. 1998, 14, 105-117. [CrossRef]

(C) 2020 by the authors. Licensee MDPI, Basel, Switzerland. This article is an open access article distributed under the terms and conditions of the Creative Commons Attribution (CC BY) license (http://creativecommons.org/licenses/by/4.0/). 